\title{
Polyrhythmic Organization of Coupled Nonlinear Oscillators
}

\author{
Gerold Baier ${ }^{1}$, Thomas Hermann², and Markus Müller ${ }^{1}$ \\ ${ }^{1}$ Faculty of Sciences, Autonomous University of Morelos \\ Cuernavaca, Morelos, Mexico \\ \{baier;muellerm\}@servm.fc.uaem.mx \\ ${ }^{2}$ Neuroinformatics Group, Bielefeld University, Bielefeld, Germany \\ thermann@techfak.uni-bielefeld.de
}

\begin{abstract}
We study the rhythmic organization of coupled nonlinear oscillators. If oscillators with non-identical internal frequency are coupled they generate a great variety of periodic and chaotic rhythmic patterns. Sonification of these patterns suggests their characterization in terms of polyrhythms: each oscillatory unit subdivides "measures" of equal or varying length differently. For the case of two coupled oscillators the organization of these polyrhythms is exemplified as a function of the internal frequency ratio and the coupling strength. Some sonification strategies are presented which aid to detect complex rhythmic relationships between oscillators. The results may be of importance for the analysis of complex multivariate time series like human EEG.
\end{abstract}

\section{Introduction}

Rhythm can be defined as a non-random temporal sequence of discrete events. In our context, "nonrandom" means that there exists some deterministic generating algorithm for the temporal sequence and, as a consequence, that there are at least short-term correlations between successive events. By polyrhythm we refer to the coexistence of two or more rhythms that are dependent on each other but do not share a common denominator in their isochronous patterning (see e.g. [1]). This means that, for instance, they divide a measure with a noninteger ratio of their number of beats. Their mutual dependence implies a finite coupling between the participating rhythms (i.e. they are not free-running).

The musical concept of polyrhythm (for a definition and examples see [2]) expresses that two rhythms that are neither in synchrony nor simple multiples of each other, can still be correlated. The common situation is that i) both rhythms are periodic (each is based on an isochronous pulse train), ii) the number of pulses per period, $p$ and $q$, have some non-integer ratio $p / q(p, q \in \mathbf{N}$, where $p>q$ ), and iii) the communication between the rhythms is expressed in periodic coincidences of pulses. From a theoretical point of view there is no reason to exclude irregular rhythms from this definition, if only they follow a fixed generating rule. The only difference to the periodic case would be that the coincidences of pulses occur at irregular intervals. Here we permit this generalization and discuss the concept of polyrhythm in the context of nonlinear dynamical systems.

We provide some background about self-organized oscillations and then study a model of linearly and reversibly coupled nonlinear oscillators. This model is a prototype used for the description of rhythmic processes in biological systems like heartbeat, neural firing, and hormone secretion among many others (a readable introduction can be found in [3]). The basis for our discussion is that each oscillator has a well-defined internal frequency (i.e., when there is no coupling between oscillators) and thereby generates a periodic sequence of "events". Sonification of the rhythms resulting from the introduction of finite coupling demonstrates that the concept of polyrhythm can be applied naturally when the rhythmic patterns are complex. We argue that this might be used to characterize complex rhythmic phenomena in biological systems (like cardiac arrhythmias and neural firing patterns) but also as a general tool in time series analysis.

\section{The Model}

We analyze the dynamics of a prototypic system of coupled nonlinear oscillators. The system consists of two FitzHugh-Nagumo (FHN) models and is given by the following set of ordinary differential equations:

$$
\begin{aligned}
& \frac{d X_{1}}{d t}=X_{1}\left(a-X_{1}\right)\left(X_{1}-1\right)-Y_{1}+I+D\left(X_{2}-X_{1}\right) \\
& \frac{d Y_{1}}{d t}=b\left(X_{1}-Y_{1}\right) \\
& \frac{d X_{2}}{\varepsilon d t}=X_{2}\left(a-X_{2}\right)\left(X_{2}-1\right)-Y_{2}+I+D\left(X_{1}-X_{2}\right) / \varepsilon \\
& \frac{d Y_{2}}{\varepsilon d t}=b\left(X_{2}-Y_{2}\right)
\end{aligned}
$$


where we fixed constants $a=0.1, b=0.01, c=1.2$, and $I=0.07 . D$ is the coupling constant and parameter $\varepsilon$ controls the frequency of the second oscillator relative to the first, i.e. the ratio of internal frequencies.

Originally, the FHN equation was introduced to describe the firing of squid neurons but it soon became clear that, with some modification, it may be used to describe the dynamics of different types of neurons, of heart cells, muscle cells, pancreas cells and others as well. All these cells display sudden eruptions of voltage, either spontaneously (oscillations) or in response to external perturbations (excitability). The corresponding models are able to reproduce these observations and they allow to make specific prediction that can be tested experimentally (for instance, that the transition between excitability and oscillations is sharp and not continuous).

An important feature of these models is that they may be applied at different levels of organization. Coupled oscillators like those in eq. 1 not only present a means to describe the interaction between individual biological cells but also between groups of cells if each group can be modeled to act synchronously. In the case of the heart, for example, a two-oscillator model might be adjusted to describe the electric interaction between the ventricles (the main pump) and the atria (the supplementary pump). As such, the investigation of coupled oscillators has become central for the understanding of rhythmic processes in living systems [3].

With the selected set of parameters each of the two units in eq. 1 exhibits periodic nonlinear oscillations (sometimes referred to as "self-excitation"). We refer to these oscillations as the internal rhythm of the individual unit. For $\varepsilon \neq 1$ the units display periodic oscillations with non-identical frequencies. For simplicity, in the present contribution the two oscillators are chosen to be identical otherwise. Complex rhythms are then formed by introducing coupling of oscillators, i.e. setting $D>0$.

\section{Sonification of Polyrhythms}

The possibly complex temporal organization of the system in eq. 1 strongly suggests the use of human listening for the analysis of its structure. Since we are interested in rhythms and rhythmical changes, the use of percussive sound events to directly represent the nonlinear oscillations is intuitive. The sonification examples presented and discussed in the following chapter employ percussive sounds at a clear pitch, created by additive synthesis, using a variable number of harmonics. Event parameters employed are spatial panning, pitch, onset, and level. For each oscillator one stream of events is created. Pitch and panning is used to facilitate stream separation; level is constant for both, while brightness (number of active harmonics) is driven by the inter-oscillator nearest-neighbor maxima distance, i.e. the temporal distance between a given maximum and the subsequent maximum in the other oscillator.
This simple mapping has proven ideal to perceive in particular polyrhythmic structures. The timbre mapping in addition supports the detection of rhythmic outliers (e.g. missing beats), which stand out in the display due to their influence on inter-oscillator event distances. This enables us to analyze the time series for rhythmical irregularities even at time compressions that are beyond the range where human listeners can clearly discern rhythm.

It is certainly possible to choose more elaborate sounds than we did, yet we believe that the rather canonic choice presented here is sufficient to qualitatively understand the system's basic rhythmic organization.

\section{Results}

We define the rhythm of eq. 1 as the temporal pattern of all suprathreshold oscillations. There are two trivial extremes: On the one end, for $D=0$ the units are independent and not part of a bigger system. In this case it is sufficient to study each unit in isolation and it is preferable not to speak of a common rhythm. On the other end, for strong enough coupling the two oscillators completely synchronize and provide the same time series. In this case the two sources are indistinguishable and the result is a single rhythm on the level of the whole system. If however finite reversible coupling is introduced that does not succeed to synchronize the oscillators, the formerly independent rhythms start to interact and influence each other. This creates non-trivial rhythmic patterns.

Fig. 1 schematically shows the generic organization of the main periodic (poly-)rhythms in eq. 1 in an octave of frequency mismatch. The parameter region $0.5<\varepsilon<1.0$ does not contain integer ratios of the internal frequencies. Nevertheless the generic integer rhythms $1: 1$ and $2: 1$ invade the region from both ends. For small values of coupling constant $D$, quasiperiodic oscillations dominate (gray areas) interrupted by the so-called Arnol'd tongues of complex periodic modes that terminate at the rational values of $\varepsilon$ for $D=0$. The width of these tongues grows as $D$ increases in accordance with the Arnol'd picture but from $D \approx 0.0015$ bifurcations create more complex behaviors (e.g. period-doubling on the lower right edge of the 2:1 tongue). Note that such differentiation is omitted in the figure.

The polyrhythms (only three of which are included in Fig. 1) start at the exact values of frequency ratio for $D=0: 3: 2$ at $\varepsilon=0.666 \ldots, 4: 3$ at $\varepsilon=0.75,5: 4$ at $\varepsilon=0.8$, and so on. For values up to $D \approx 0.01$ the respective tongue widths grow. For higher coupling strength, the tendency to synchronize the spiking in both units diminishes the widths of all tongues except 1:1, which grows considerably. In addition, there are other complex periodic rhythms (like the period-doubled 6:4 rhythm at $\varepsilon=0.45, D=0.005$, or a $8: 5$ rhythm at $\varepsilon=0.73, D=0.0045$ ); quasiperiodic; and deterministic chaotic rhythms. These are found in the gray areas and are not differentiated in the figure. 


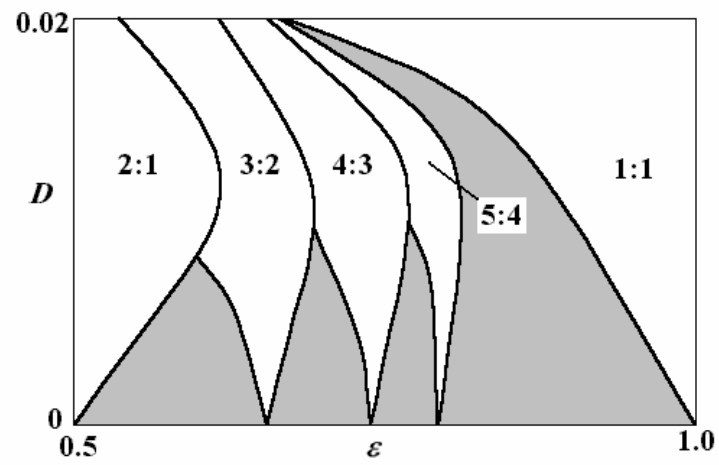

Figure 1: Schematic drawing of main periodic rhythms in eq. 1 in the $\varepsilon / D$ parameter plane. For simplicity, some details are omitted.

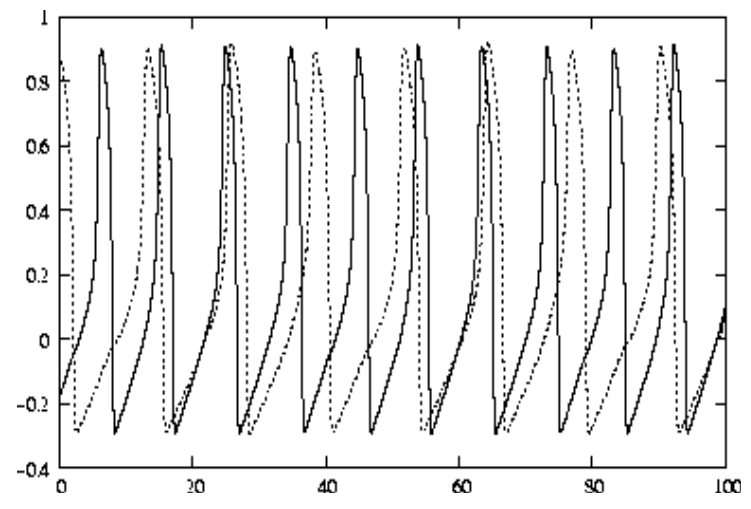

Figure 2: Time series of a periodic polyrhythm (frequency ratio $4: 3$ ) in eq. 1 with $\varepsilon=0.75$, $D=0.005$. Shown are the $X$-variables of oscillator 1 (solid line) and 2 (dotted line).

Fig. 2 shows the two time series of the two $X$ variables of the $4: 3$ rhythm as a function of time (for $\varepsilon=0.75, D=0.005$ ). The faster firing of oscillator 1 (solid line) produces nearly equal intervals between maxima of the oscillations and every fourth maximum almost coincides with a maximum of oscillator 2 . For oscillator 2 a pattern of three events is repeated where two of the distances between maxima are comparable but the third distance is somewhat larger. Sonification 1 exemplifies this polyrhythm in a stereo setting [4]. The frequencies assigned to the events in each channel have a ratio of $4: 3$ according to the internal frequencies of the two oscillators.

Compared to the parameters in Fig. 2 a slight modification in frequency ratio led to the result displayed in Fig. 3. Here, the rhythm is asymptotically aperiodic and shows a broadening of the Fourier spectrum (as compared to Fig. 2). Calculation of the spectrum of
Lyapunov characteristic exponents (LCEs) yields one positive, one zero and two negative exponents. The positive exponent indicates deterministic chaos. The time series of the slower unit is characterized by "missing beats", i.e. subthreshold oscillations with small amplitude caused by perturbations that prevent the rising of the amplitude when the spike is due (according to internal frequency). The rhythm is unable to maintain a single repetitive pattern. However, it still shows the 4:3 pattern occasionally. Due to the vicinity of the chosen parameters to the $4: 3$ region in parameter space the $4: 3$ sequence of spikes recurs from time to time and could be called a characteristic "motif" of the chaotic rhythm. This can be heard in Sonification S2.

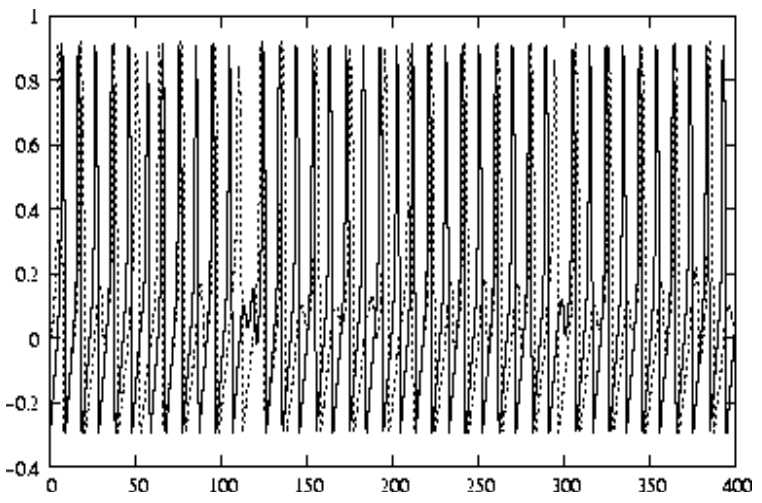

Figure 3: Time series of a chaotic rhythm in eq. 1 with $\varepsilon=0.74, D=0.005$. Shown are the $X$ variables (as in Fig. 1)

For lower values of constant offset $I$ in eq. 1 the picture is not as simple as given in Fig. 1. In particular, near the vicinity of the onset of self-excitation there are complications due to dominant chaotic attractors. E.g. at $I=0.0605, \varepsilon=0.6445, D=0.005$ the two units produce bursts of spikes. Strangely, between such bursts there are no spikes in neither unit. It appears as if the spiking in the two units were switched on and off irregularly by some external factor (Sonification S3).

As a simple extension of the above results we calculated a system composed of three identical, symmetrically coupled units. In that case we deal with two parameters, $\varepsilon_{1}$ and $\varepsilon_{2}$, for the control of internal frequencies in oscillators two and three, respectively. This way we can realize a more complex polyrhythm like, for example, 5:4:3. A time series of the three $X$ variables in that case is shown in Fig. 4. Sonification example S4 displays how the three units divide a "measure" (the time between two synchronizations of all three units) into 5,4 , and 3 beats, respectively. The three-dimensional parameter space $D / \varepsilon_{1} / \varepsilon_{2}$ has (except for $D$ close to zero) a highly complex organization of periodic, complex periodic, quasi-periodic, and deterministic chaotic rhythms. An example of a chaotic 
rhythm between three oscillators is provided in Sonification 5. While the fastest oscillator maintains a constant pulse, the other two oscillators appear to impede each other leading to unexpected rhythmic variations. Given the completely deterministic nature of the source of the signal, the sonification reveals a surprising richness in their interaction pattern.

It is known that human listeners are not only sensitive to the perception of rhythms but also to the perception of changes in rhythm. The following sonification examples make use of this skill. For this purpose the system of two FHN oscillators eq. 1 is integrated with a linearly changing frequency ratio $\varepsilon$. This is equivalent to a horizontal line in Figure 1.

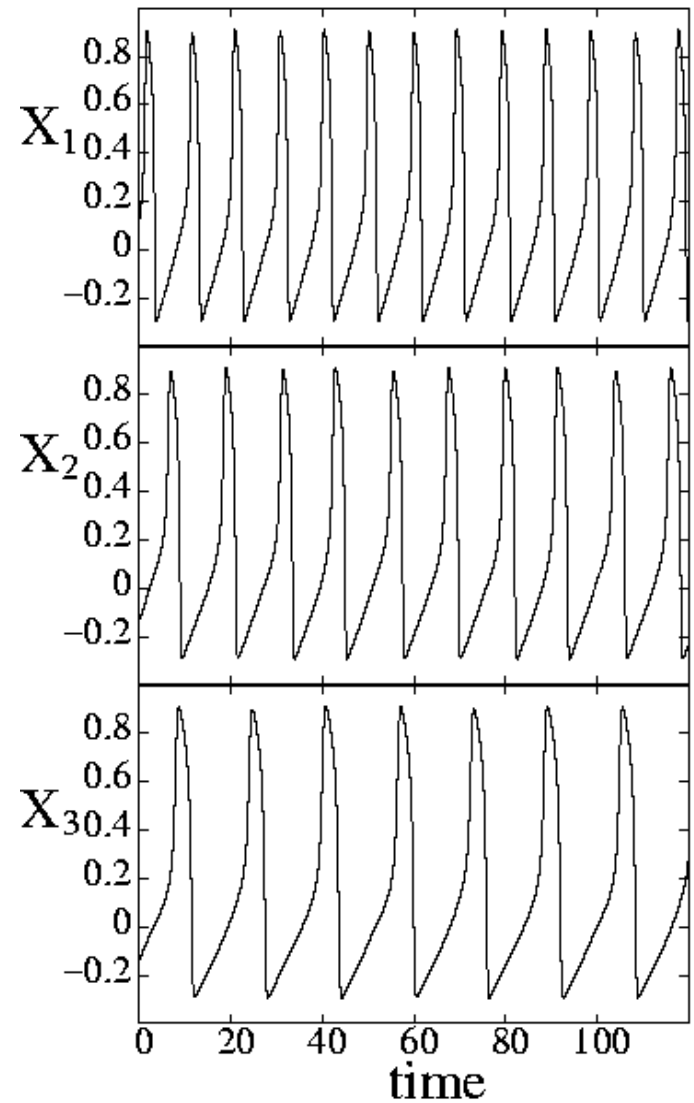

\section{Figure 4: Time series of $X$-variables for a periodic 5:4:3 polyrhythm in a system of 3 coupled FHN oscillators.}

In the first example, S6, the simulation starts on the left edge of Fig. 1 from point $(D=0.015, \varepsilon=0.5)$ with a sonification of the 2:1 rhythm and then changes $\varepsilon$ from 0.5 to 1.0 while keeping $D$ constant. Along this line it crosses, one by one, the borders to various polyrhythms. We perceive the constant beats of oscillator 1 whereas the rate of oscillator 2 increases. The rhythmical changes are immediately obvious. They are not continuous but proceed in well-defined steps. Due to the finite speed of parameter variation and the model's inertia, complex polyrhythms that occupy only a narrow window along the chosen parameter line are not resolved numerically and cannot be heard in the example.

Sonification example S7 starts from the same point $(D=0.015, \varepsilon=0.5)$ towards the right edge of Fig. 1 but extends the duration to 1 minute. Listening to the sound, not only the polyrhythmic regimes can be better distinguished, also another feature can be perceived: within a polyrhythmic regime the "meter" continuously slows down. Such features remain easily unattended in time series plots, yet they are immediately obvious in the sonification, and demonstrate the utility of sonification in detecting qualitative behavior in studying dynamic systems in general.

\section{Discussion}

Coupled nonlinear oscillators are able to display polyrhythmic firing patterns if their internal frequencies are not close to an integer ratio $\mathrm{p} / \mathrm{q}$, with $\mathrm{p}>\mathrm{q}$. While such polyrhythmic phenomena have been discussed a long time ago as a possible explanation of the so-called Wenckebach rhythms in cardiology [5], we are not aware of a systematic investigation of polyrhythms in the context of other biological systems, notably the firing patterns of coupled neurons. In particular, auditory nerve fibers are known to possess clearly differentiated internal frequencies [6].

The FHN equation is a prototypic model of a type 2 excitable system (finite frequency at the onset of selfoscillation). It can be adjusted to describe a variety of waveforms found experimentally in excitable cells and tissues. The parameters for eq. 1 were chosen such that each model unit displays periodic nonlinear action potentials. All deviations from this simple periodic behavior are a consequence of the mutual coupling of the oscillators. As oscillator 1's internal frequency is always higher than that of oscillator 2 for $\varepsilon<1.0$, its time series is normally continuously spiking whereas missing beats and subthreshold oscillations are found in oscillator 2 . However, in chaotic and intermittent regimes missing beats are also possible in the first oscillator (e.g. Sonification S3).

The definition of rhythm presented in the introduction is based exclusively on the temporal order of the data and thus on measurable physical properties. Such an approach is essential because the sonifications are supposed to reveal the dynamic patterns of the data. This is opposite to studies of rhythm that use a definition based on the perceptual response of the listener [1]. The results of these psychological studies will have to be taken into account, however, when it comes to the correct interpretation of the perceptions. Even though for example clear preferences in the (temporal) grouping of sound events can be found, the perception of rhythm varies strongly among listeners when complex pattern like polyrhythms are involved [7]

Previous sonifications of rhythms in (non-excitable) ordinary differential equations have focused on the 
aspect of limited irregularity of deterministic chaos [8]. There it was first argued that listening to these rhythms forms an important approach for the analysis of irregular but non-random temporal events. A similar argument can be derived from the present numerical studies. Comparing sonifications of the periodic and chaotic rhythm (S1 and S2, see Figs. 2 and 3) the occurrence of a common motif makes the relationship between the rhythms obvious. As in the case of most published studies that offer numerous graphical representations to familiarize the reader with rhythmic dynamics (like e.g. [3]), it is now recommended to perform extensive explorations of the parameter space to obtain a sophisticated aural representation of the rhythmic richness and relationships. Whereas in the simplest case of 2 coupled oscillators this amounts to basically repeating in a second sensory mode an already completed analysis, we conjecture that the aural representation may be superior in the more complex cases of three or more coupled oscillators (Sonification S4) and as such may provide new insights that were not yet obtained from the traditional representation.

A particularly promising strategy is to combine the auditory display with animated visualization, for instance of the time series or some truncated form of it. From our preliminary experience it is likely that the visual display can be coupled to the sonifications without conflict between the media. If in addition the user could interact with a graphical display (e.g. navigate a parameter vector in a plot like Fig. 1, the two perceptual modes may even mutually fertilize the understanding. A multimedia approach will then accelerate and deepen insight into the polyrhythmic quality of the dynamics.

It is in the more complex cases that the exploitation of additional features of the time series and their representation in sound parameters becomes indispensable. For example, in a system of 3 coupled FHN oscillators recently so-called hyperchaotic behavior was observed [9]. Hyperchaos is a deterministic process that is identified by the presence of 2 or more positive Lyapunov exponents as compared to only 1 for simple chaos (see [10] for technical details). A preliminary study of such hyperchaotic systems composed of oscillators with different internal frequencies revealed intriguing rhythmic patterns [11] but so far a deeper understanding is lacking. Rhythmic sonification as demonstrated in the present contribution offers a route to such understanding.

Immediate application of rhythmic sonification is possible in cardiology where complex rhythmic activity and rhythmic transitions are known to precede the lifethreatening state of fibrillation. An important advantage of sonification in this case is that it can implement knowledge obtained from electrocardiographic recordings, e.g. it might assign the individual contributions of atria and ventricles into separate sound sources.

Finally it should be mentioned that the concept does not only apply for time series where the rhythmic behavior is obvious. An important example is human electroencephalogram (EEG), where signals are noiselike and hard to quantify in terms of rhythms but where temporal correlations nevertheless are suspected to be present, if only transiently (for a sonification of the transition from normal to epileptic behavior see [12]). For such cases recently a new method of analysis was proposed that extracts information in the form of rhythmic spike sequences [13]. The process works similar to groups of sensory neurons that convert analog signals into temporal sequences of action potentials. In both cases the outputs of the analysis are rhythmic according to our definition if they are non-random. To apply the proposed concept of polyrhythm to EEG data one would have to decompose the signals recorded at different positions on the scalp and then sonify them with the techniques given above. A first attempt was presented in [14] for the transition from normal to epileptic behavior but further research is necessary to clarify the differences between complex rhythms (like the hyperchaotic solutions mentioned) and truly random sequences of events.

\section{Acknowledgements}

This work was supported by CONACyT, Mexico (project no. 40885-F). G.B. thanks Sven Sahle, EML Research, Heidelberg, for discussion.

\section{List of Sonifications}

All sonifications can be found in the following webpage: http://www.techfak.unibielefeld.de/ thermann/projects/index.html

S1: A 4:3 polyrhythm with parameters as in Fig. 2.

S2: A chaotic polyrhythm with parameters as in Fig. 3 in two different speeds.

S3: An intermittent polyrhythm with parameters $I=0.0605, \varepsilon=0.6445, D=0.005$.

S4: A periodic polyrhythm in a three oscillator system with internal frequencies set to $5: 4: 3$ and $D=0.01$ (other parameters as in eq. 1).

S5: A chaotic polyrhythm in a three oscillator system with internal frequencies set to 1.0:0.9:0.55 and $D=0.0032$ (other parameters as in eq. 1). Duration a) 45 sec; b) $15 \mathrm{sec}$; and c) $5 \mathrm{sec}$.

S6: Sequence of rhythms in eq. 1 as parameter $\varepsilon$ is continuously varied from 0.5 to 0.99 .

S7: Sequence of rhythms as in S6 but with total duration $1 \mathrm{~min}$.

\section{References}

[1] S. Handel, Listening, MIT Press, Cambridge, 1989, chapter 11.

[2] http://www.xs4all.nl/ marcz/Polyrhythm.html 
[3] L. Glass, M. Mackay, From Clocks to Chaos, Princeton University Press, 1988.

[4] http://www.techfak.uni-bielefeld.de/ thermann/projects index.html

[5] K.F. Wenckebach. Arrhythmia of the Heart: a Physiological and Clinical Study. Green, Edinburgh, 1904.

[6] see e.g. W.L. Gulick, G.A. Geschieder, R.D. Frisina, Hearing - Physiological Acoustics, Neural Coding, and Psychoacoustics. Oxford University Press, New York 1989.

[7] S. Handel, Using Polyrhythm to Study Rhythm, Music Perception 1, 465 (1984).

[8] G. Baier and S. Sahle, Listening to Chaos: the Aural Representation of Irregular Rhythms. In: H.H. Diebner, T. Druckery and P. Weibel (eds.), Sciences of the Interface, Genista Verlag, Tübingen (2001), p. 255. Sonifications at: http://basic-research.zkm.de:8080/basic_research/projekte /?top=vertonung

[9] G. Baier and M. Müller, Excitable Chaos in Diffusively Coupled FitzHugh-Nagumo Equations. Rev. Mex. Fís. 50, 422 (2004).

[10] G. Baier and M. Klein (Eds.), A Chaotic Hierarchy. World Scientific, Singapore 1991.

[11] G. Baier and S. Sahle, Spatio-Temporal Patterns with Hyperchaotic Dynamics in Diffusively Coupled Biochemical Oscillators. Discrete Dynamics in Nature and Society 1, 161 (1997)

[12] S. Sahle and G. Baier, track 16 of the CD accompaning G. Baier, Rhythmus (in German), Rowohlt Verlag, Reinbeck, 2001. Also c.f. "Listening to epilepsy" on the webpage http://www.cerebraldynamics.org

[13] G. Baier, R. Leder, P. Parmananda, Human Electroencephalogram Induces Transient Coherence in Excitable Spatio-temporal Chaos, Phys. Rev. Lett. 84, 4501 (2000). G. Baier and M. Müller, The Nonlinear Dynamic Conversion of Analog Signals into Excitation Patterns, Phys. Rev. E 70, 037201 (2004).

[14] G. Baier and T. Hermann, The Sonification of Rhythms in Human Electroencephalogram. Proceedings of ICAD 2004, July 6-9, Sydney (2004). See also: T. Hermann, G. Baier and M. Müller, Polyrhythm in the Human Brain. Proceedings of ICAD 2004, July 6-9, Sydney (2004). 